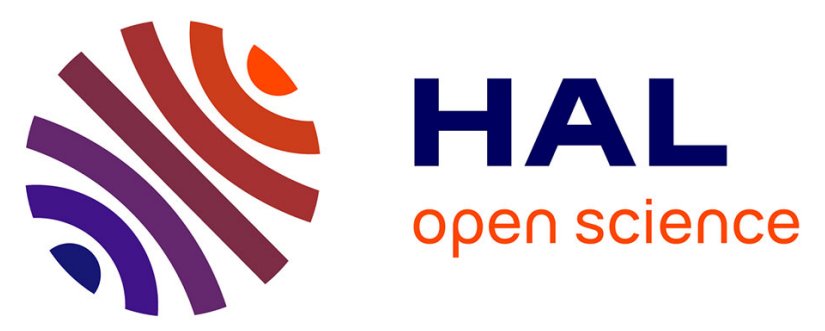

\title{
Dramatic Slowing Down of Oil/Water/Silica Contact Line Dynamics Driven by Cationic Surfactant Adsorption on the Solid
}

Gaëlle Rondepierre, Franz de Soete, Nicolas Passade-Boupat, François Lequeux, Laurence Talini, Laurent Limat, Emilie Verneuil

\section{To cite this version:}

Gaëlle Rondepierre, Franz de Soete, Nicolas Passade-Boupat, François Lequeux, Laurence Talini, et al.. Dramatic Slowing Down of Oil/Water/Silica Contact Line Dynamics Driven by Cationic Surfactant Adsorption on the Solid. Langmuir, 2021, 37 (5), pp.1662-1673. 10.1021/acs.langmuir.0c02746 . hal-03189529

\section{HAL Id: hal-03189529 \\ https://hal.science/hal-03189529}

Submitted on 4 Apr 2021

HAL is a multi-disciplinary open access archive for the deposit and dissemination of scientific research documents, whether they are published or not. The documents may come from teaching and research institutions in France or abroad, or from public or private research centers.
L'archive ouverte pluridisciplinaire HAL, est destinée au dépôt et à la diffusion de documents scientifiques de niveau recherche, publiés ou non, émanant des établissements d'enseignement et de recherche français ou étrangers, des laboratoires publics ou privés. 


\title{
Dramatic Slowing Down of Oil/Water/Silica Contact Line Dynamics Driven by Cationic Surfactant Adsorption on the Solid
}

\author{
Gaëlle Rondepierre, Franz De Soete, Nicolas Passade-Boupat, François Lequeux, Laurence Talini, \\ Laurent Limat, and Emilie Verneuil*
}

\begin{abstract}
We report on the contact line dynamics of a triplephase system silica/oil/water. When oil advances onto silica within a water film squeezed between oil and silica, a rim forms in water and recedes at constant velocity. We evidence a sharp (three orders of magnitude) decrease of the contact line velocity upon the addition of cationic surfactants above a threshold concentration, which is slightly smaller than the critical micellar concentration. We show that, with or without surfactant, and within the range of small capillary numbers investigated, the contact line dynamics can be described by a friction term that does not reduce to pure hydrodynamical effects. In addition, we derive a model that successfully accounts for the selected contact line velocity of the rim. We further demonstrate the strong increase of the friction coefficient with surfactant bulk concentration
\end{abstract}
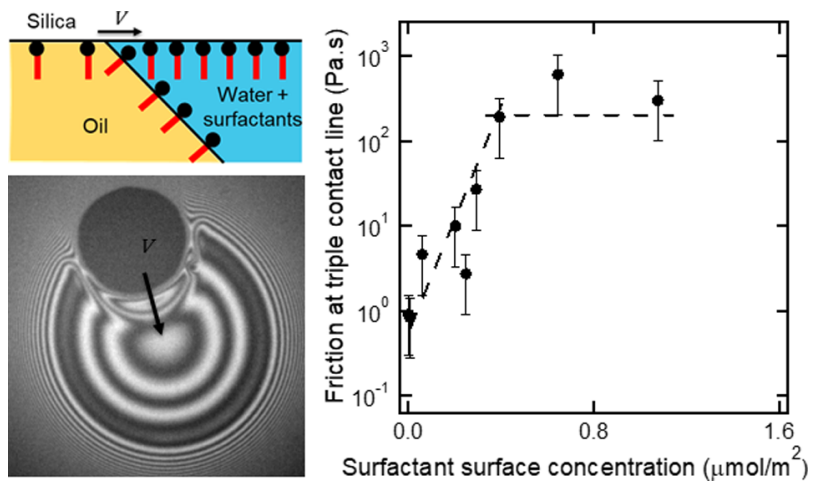
results from the strongly nonlinear adsorption isotherm of surfactants on silica. From the variations of the friction coefficient and spreading parameter with surface concentration, we suggest a picture in which the part of the adsorbed surfactants that are strongly bound to the silica interface is trapped under the oil droplet and is responsible for the large increase in line friction.

\section{INTRODUCTION}

The impact of surfactants on the wettability of solid substrates is of interest to many industries, ranging from industrial coating processes to controlled spraying of active agents, in agriculture for example. As such, it has been studied extensively. $^{1-8}$ In literature, the wettability of surfaces by surfactant solutions was often characterized through measurements of equilibrium contact angles $\alpha_{\mathrm{e}}$ of droplets of surfactants solutions in air: the larger the angle, the lower the wettability. These macroscopic characterizations were interestingly related to the arrangement of surfactants on the solid surface depending on their concentration. ${ }^{9-13}$ Beyond the static case, the wetting dynamics of surfactants solutions is expected to involve complex interplay between transfers at both liquid and solid interfaces, liquid flows, and possibly specific behaviors at the contact line due to surfactants' adsorption or desorption. Over the past decades, the wetting dynamics of pure liquids over solid substrates, in air or in another liquid, without any surfactants, has been extensively studied both experimentally and theoretically. ${ }^{14-22}$ A moving contact line exhibits a dynamic contact angle $\alpha$, which differs from its equilibrium value due to dissipation, and depends on the contact line velocity. Spontaneous wetting is agreed to be driven by the gain in surface energy, $\gamma\left(\cos \alpha_{\mathrm{e}}-\cos \alpha\right)$, while several dissipative processes have been thought of, which involve very different lengthscales. ${ }^{17}$ Viscous dissipation in the spreading liquid, first, was modelled in the Stokes framework, using lubrication approximation for small-to-moderate contact angles $^{19,20,23}$ and is expected to apply at distances to the contact line ranging from a nanometer-sized length to the macroscopic system size. Second, at a microscopic scale, the contact line motion has been modeled in terms of dissipative molecular displacements between adsorption sites on the solid surface, where the molecular jumps are associated to a barrier activation energy. ${ }^{18,22,24}$ Experimentally, both models were shown to describe experimental data depending on the range of contact line velocities $V$. More precisely, a nondimensional capillary number is usefully defined as $\mathrm{Ca}=\eta V / \gamma$ where $\gamma$ is the liquid surface tension and $\eta$ is the viscosity. ${ }^{23}$ Hence, in most liquids, viscous dissipation was shown to control the wetting dynamics at large Ca numbers. Conversely, at smaller velocities or $\mathrm{Ca}$ numbers, the molecular kinetic theory was found to successfully describe experimental data, ${ }^{25-27}$ even 
though some authors suggested other origins for friction at the contact line. $^{28-30}$ A model combining both hydrodynamic dissipation and molecular theory was also developed to describe intermediate contact lines velocities and was successfully found appropriate in some cases. ${ }^{21,31,32}$ However, this picture does not describe the wetting dynamics for all systems. $^{31-33}$

Adding surfactants to the spreading liquid was shown to induce an important increase of the wetting speed of surfactant solutions on solid surfaces. This phenomenon is called superspreading and was observed with surfactants in the advancing phase. ${ }^{5,7,34,35}$ The case of receding surfactant solutions, especially in a two-liquid problem, has been much less studied. ${ }^{1,8}$ In all cases, transfers of surfactants in the liquid phase and toward the air/liquid interface are regarded as the determining step controlling the contact line dynamics, ${ }^{6,36,37}$ with possible surfactant transport along the liquid/air interface through Marangoni effects. ${ }^{1}$ Yet, no clear picture of the effect of surfactants adsorbed to the solid interface on the wetting dynamics can be found in literature.

In the present work, we offer to investigate the dewetting dynamics of surfactant solutions at low capillary numbers, with surfactants added to the receding phase, and in a two-liquid situation. The low capillary number regime is expected to enhance the effect of surfactants adsorbed to the solid interface. To gain accurate knowledge of the behavior of adsorbed surfactants, we carefully characterized its adsorption to the solid at equilibrium. The ternary system studied here is silica as the solid, aqueous solutions of surfactants as the receding phase (hereafter called water), and oil as the advancing phase, as depicted in Figure 1. This system is of

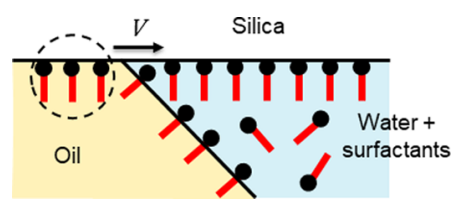

Figure 1. Sketch of a triple contact line between silica, water, and oil. Water recedes at velocity $V$. Water-added surfactants adsorb at the oil-water and water-silica interfaces. Dotted circle: As water recedes, surfactants may remain adsorbed at silica/oil interface or desorb from silica (either partially or totally).

particular interest to applications where membranes are used to separate oil and water in waste water management processes. ${ }^{38-40}$ By experimentally studying the receding dynamics of aqueous solutions of surfactants on silica and in oil, we will determine the respective effects of surfactants adsorbed and transferred to the three interfaces at stake: solid/ water, solid/oil, and water/oil. The contact line dynamics will be analyzed both in terms of surface energy gain, viscous dissipation, and molecular friction at the contact line. In particular, since surfactants are in the receding phase only (Figure 1), the question of their trapping at the solid/oil interface or their possible desorption at the contact line will be tackled.

\section{EXPERIMENTAL SECTION}

Materials. The two liquids were chosen to be immiscible: water and dodecane (Sigma-Aldrich, purity $>99 \%$ ). To guarantee a reproducible physico-chemistry, the solid substrates were uncoated UV-fused plano-convex silica lenses (SPX031) with focal lens $500 \mathrm{~mm}$ from Newport. To ensure an optimal cleanliness of the surface, the following procedure was used prior to each experiment. As received, the lenses were submitted to a heating cycle at $620^{\circ} \mathrm{C}$ for $15 \mathrm{~min}$ in a furnace, to burn any organic impurity or surface coating. They were further dusted out under a nitrogen flux. Right before the experiment, each lens was exposed to an ozone atmosphere for $15 \mathrm{~min}$ and then immersed immediately into the work solution to avoid any contamination.

The surfactant was chosen with a good ability to adsorb on the negatively charged silica and very low solubility in oil. Consequently, we chose hexadecyltrimethylammonium bromide (CTAB) from Sigma-Aldrich (purity $>96 \%$ ), which meets these criteria. ${ }^{41}$ It was dissolved as received in deionized water with an added background concentration of $10^{-4} \mathrm{~mol} \cdot \mathrm{L}^{-1}$ of $\mathrm{NaCl}$ to set the value of the ionic strength in the water phase. The $\mathrm{CTAB}$ concentration $[\mathrm{CTAB}]$ was varied between $6 \cdot 10^{-7}$ and $4 \cdot 10^{-3} \mathrm{~mol} / \mathrm{L}$. In this range of concentration, the viscosity $\eta$ of the aqueous solutions is found constant $\eta=0.89 \mathrm{mPa} \cdot \mathrm{s}$. The viscosity of dodecane is $\eta_{\mathrm{oil}}=0.75 \mathrm{mPa}$. s.

Adsorption at the Liquid/Liquid Interface. Tensiometry experiments were conducted to determine the interfacial tension of $\mathrm{CTAB}$ solutions with dodecane as a function of the concentration. Experimental details and results are shown in the Supporting Information. The interfacial tension decreases with bulk surfactant concentration until it reaches a plateau at the critical micellar concentration (CMC). ${ }^{42}$ We find $\mathrm{CMC}=0.9 \mathrm{mmol} \cdot \mathrm{L}^{-1}$, in agreement with values from literature for $\mathrm{CTAB}$ with no added salt. ${ }^{41,43}$ Data from literature show that sorption equilibrium between $\mathrm{CTAB}$ in the bulk at concentration $C$ and at the oil/water interface (surface concentration $\Gamma_{i}$ ) is well described by a Langmuir isotherm and Gibbs equation. ${ }^{41}$ At saturation, the surface concentration is denoted $\Gamma_{i}^{\infty}$ and measured at $1.8 \mu \mathrm{mol} \cdot \mathrm{m}^{-2}$, in agreement with existing data on CTAB at air or oil interfaces. ${ }^{43-45}$ For the dodecane/pure water interfacical tension, we measure $\gamma=41.9 \mathrm{mN} \cdot \mathrm{m}^{-1}$ and above CMC, $\gamma=7 \mathrm{mN} \cdot \mathrm{m}^{-1}$. We also checked, by fast swelling and deswelling of the oil drop, that the transport of surfactants at the oil water interface is governed by diffusion (data not shown).

Adsorption at the Solid/Liquid Interface. We characterized the adsorption of $\mathrm{CTAB}$ at the water-silica surface especially because it strongly depends on the nature and quantity of added salt, ${ }^{46,47}$ using the depletion method described in the Supporting Information.

The adsorption isotherm, namely, the variations of the surface concentration $\Gamma$ with $\mathrm{CTAB}$ concentration, is shown in Figure 2. The amount of $\mathrm{CTAB}$ adsorbed on silica increases with bulk concentration, or surfactant chemical potential, in three steps as follows: slow increase below $\Gamma^{*}$, step-like increase above $\Gamma^{*}$, and then saturation to a plateau value $\Gamma^{\infty}$ of $2.4 \mu \mathrm{mol} \cdot \mathrm{m}^{-2}$ similar to that measured in literature. ${ }^{46,48-50}$ The shape of the isotherm results from

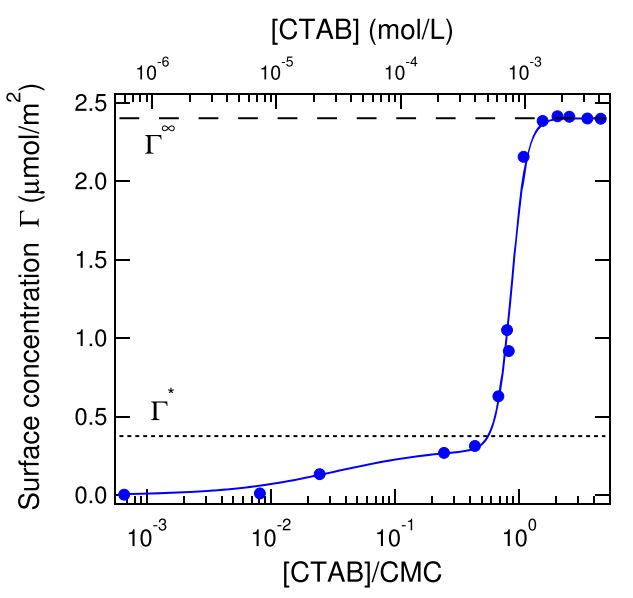

Figure 2. Adsorption isotherm of $\mathrm{CTAB}$ on silica. Dotted line: $\Gamma^{*}=$ $0.37 \mu \mathrm{mol} \cdot \mathrm{m}^{-2}$. Dashed line: $\Gamma^{\infty}=2.4 \mu \mathrm{mol} \cdot \mathrm{m}^{-2}$. Full line: fit to eq 1 with $k_{1}=30, k_{2}=2.7$, and $n=8.5$. 
the arrangement of the surfactants on the solid surface, and the present picture emerging from literature is the following: ${ }^{51,52}$ isolated aggregates are first detected around a bulk concentration $10^{-5} \mathrm{~mol}$. $\mathrm{L}^{-1}$. As bulk concentration further increases, monolayered-like patches of surfactants progressively form from these aggregates, grow, and finally connect to form a network, until a complete monolayer is obtained at larger concentration. Further increase in bulk concentration leads to the formation of bilayer-like patches on top of the underlying monolayer until a complete bilayer covers the substrate, the top layer of which possibly being less dense than the monolayer. The complete bilayer corresponds to the plateau value $\Gamma^{\infty}$. In this picture, the sharp increase of $\Gamma$ is attributed to adsorption mechanisms with low energetical cost compared to the electrostatically driven adsorption of isolated positively charged $\mathrm{CTA}^{+}$ions onto the negatively charged silica surface. Such mechanisms may be the tail-to-tail surfactants aggregation typically involved in the growth of bilayer-like patches on top of the monolayer ${ }^{41}$ or earlier surfactants adsorbing with their counter-ion during the growth of monolayer-like patches. ${ }^{5,51}$ Altogether, the adsorption isotherm in Figure 2 reveals that $\mathrm{CTAB}$ adsorbs through strong interactions at low concentration, until a surface coverage denoted $\Gamma^{*} \sim 0.4 \mu \mathrm{mol} / \mathrm{m}^{2}$. Above $\Gamma^{*}$, the step-like isotherm reveals that further adsorption involves weaker interactions so that these additional surfactants are expected to be very labile.

Experimental determinations of the adsorption isotherm of surfactants on solid surfaces from literature ${ }^{53}$ allowed the derivation of the empirical eq 1

$$
\Gamma=\frac{\Gamma^{\infty} k_{1} C\left(1 / n+k_{2} C^{n-1}\right)}{1+k_{1} C\left(1+k_{2} C^{n-1}\right)}
$$

where $\Gamma^{\infty}$ is the surface concentration at saturation, and $C=$ $[\mathrm{CTAB}] / \mathrm{CMC}$ is the concentration relative to the CMC. Eq 1 was fitted to our experimental data $\Gamma(C)$ in Figure 2 with $k_{1}, k_{2}$, and $n$ as fitting parameters. It was later used to compute $\Gamma$ at all concentrations.

Squeezed Droplet Experiments. A homemade setup ${ }^{54}$ was designed to observe the water/oil/silica contact line dynamics both within a water film trapped between a squeezed oil drop and silica and at the edge of the oil drop when it spreads on silica within water. A schematic representation is given in Figure 3, and a detailed
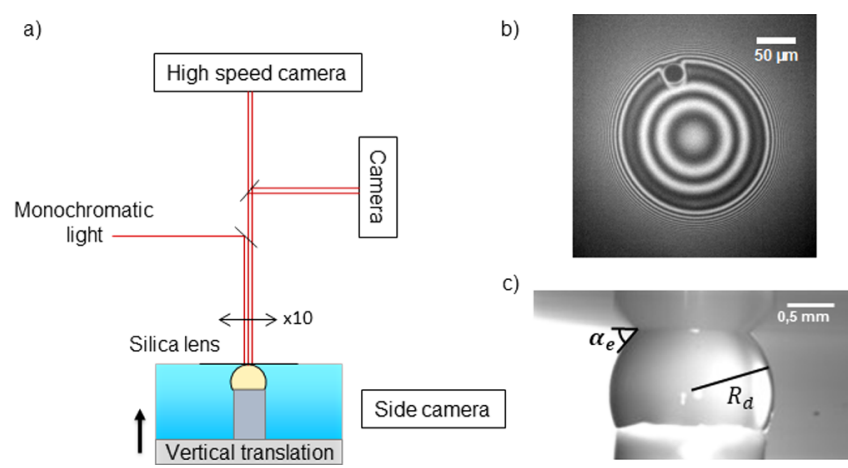

Figure 3. (a) Experimental setup. (b) Interference microscopy image within the squeezed water film between oil and silica. (c) Side image of the oil droplet of radius $R_{\mathrm{d}}$ immersed in water and squeezed against a silica lens. After complete dewetting of the water film, oil connects to silica in the aqueous solution at a static receding contact angle $\alpha_{\mathrm{e}}$.

description can be found in a previous work. ${ }^{54} \mathrm{~A}$ transparent PMMA tank is filled with the aqueous solution. An oil drop (Figure 3c) of controlled volume ranging from 0.5 to $1 \mu \mathrm{L}$ is created at the tip of a polymer tube (PEEK, ID $65 \mu \mathrm{m}$, OD $1.59 \mathrm{~mm}$ ) connected to a syringe pump. Its radius is denoted $R_{\mathrm{d}}$. Both the tube with the drop and the tank are set to a motorized vertical stage (PI). The silica lens is held at the water surface and set to a $10 \times$ microscope objective (Zeiss Epiplan Apochromat). As depicted in Figure 3a, observations of the lens/water/oil interfaces were made through the lens with a microscope with episcopic monochromatic illumination $(\lambda=615 \mathrm{~nm})$. A typical image is shown in Figure $3 \mathrm{~b}$ and will be discussed hereafter. Finally, millimeter-sized views of the oil droplet are imaged with a side camera (Sentech) at $10 \mathrm{fps}$ and low magnification $(2.8 \mu \mathrm{m}$ per pixel) from which the oil drop radius $R_{\mathrm{d}}$ was measured and the low velocity range was explored (Figure 3c).

The following procedure is used: First, the tank is filled with the surfactant+salt solution, an oil drop is created at the tip of the tube in the filled tank, and the silica lens is immersed in the tank also. Prior to any experiment, relaxation of the oil/water and water/silica interfaces is allowed for 30 and $15 \mathrm{~min}$, respectively. Second, the drop is squeezed toward the surface at $15 \mathrm{~mm} \cdot \mathrm{s}^{-1}$ on a distance varying between 35 and $60 \mu \mathrm{m}$ and then stopped. During the squeezing stage, water remains trapped between oil and silica. The oil/water interface is deformed in a well-known bell shape called a dimple ${ }^{4,54-57}$ that subsequently drains. Here, the squeezed water film is pressurized by the oil drop and experiences a pressure equal to the drop capillary pressure $2 \gamma / R_{\mathrm{d}}$ ranging from 20 to $120 \mathrm{~Pa}$. Depending on the concentration in surfactants, the draining water film may either stabilize or spontaneously puncture, resulting in water dewetting from the silica surface in oil. We emphasize the squeezing stage is needed to trap a water film and nucleate the dewetting of water. However, once initiated, that process is spontaneous, and its dynamics is independent of the applied pressure. The dewetting case is the scope of the present paper.

First, after dewetting of water has occurred, the equilibrium angle $\alpha_{\mathrm{e}}$ in water at the triple contact line was measured as the static receding contact angles at vanishing velocity at the edge of the drop (Figure 3c). Vanishing velocities were obtained by low-rate injection of oil in the drop. This angle typically varies between $40^{\circ}$ and $100^{\circ}$.

Second, when the squeezed water film punctures, the water film thickness profile is accurately measured over space and time and at the edge of the receding triple contact line, through optical interferometry, using the light reflected at the two interfaces and collected by the microscope. Typical microscopy images are shown in Figures $3 \mathrm{~b}$ and 4 , where the rings are equal thickness fringes and the wetting of oil on silica appears as a black circular area. Contact line velocities are measured from images recorded with framerates ranging from 10 to 13,600 frames per second, thereby allowing a wide velocity range (from $10^{-3}$ to $50 \mathrm{~mm} \cdot \mathrm{s}^{-1}$ ). To do so, a double camera system equipped with a microscope was used: a high speed camera (Photron Mini) with a resolution of $0.5 \mu \mathrm{m}$ per pixel and a $10 \mathrm{fps}$ camera (Sentech) with a resolution of $0.14 \mu \mathrm{m}$ per pixel. A typical series of interferometry images is shown in Figure $4 a-c$ for a CTAB solution at $0.001 \mathrm{CMC}$. The corresponding profile of the silica and oil/water interface is depicted in Figure 4d.

In the vicinity of the quasi-circular oil/silica contact zone, interference fringes accumulate, indicating a large thickness variation of the water film. They can be used to measure the thickness profile of the water film, in the vicinity of the contact line, as shown in Figure 4e. A dewetting rim is found to collect the water, as described in literature. ${ }^{23}$ At the oil/water/silica contact line, water connects at an angle we denote $\alpha_{\text {rim }}$ that can be measured from the thickness profiles. Typical results are plotted in Figure $4 \mathrm{f}$ at $[\mathrm{CTAB}]=10^{-3}$. CMC. The contact angle is found to be small (around $6^{\circ}$ in this case at long times), and for all aqueous solutions tested, it saturates to a steady value that will be taken as the dynamic contact angle value $\alpha_{\text {rim }}$ in the following.

Finally, the growth rate of the dewetted area $A$ is measured. Remarkably, the dewetted zone is quasi circular evidencing that the contact line velocities do not depend on the local thickness (Figure $4 \mathrm{a}-\mathrm{c})$. Typical time variations of its radius $R=\sqrt{A \pi}$ are shown in Figure 4f. A linear dependency of $R$ with time is observed for all experiments, as reported in literature. ${ }^{17,58,59}$ The slope determines the dewetting velocity of the rim denoted $V_{\text {rim }}$. Here, the sign of the dewetting velocity is chosen positive when water recedes and oil advances. 
a)

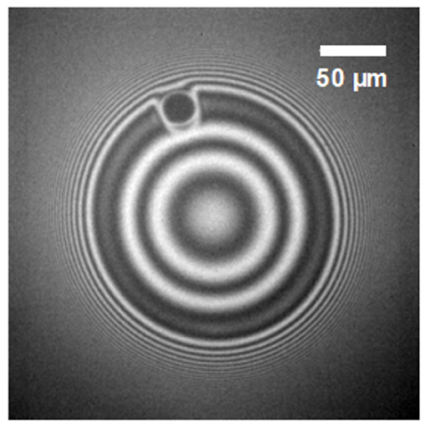

d)

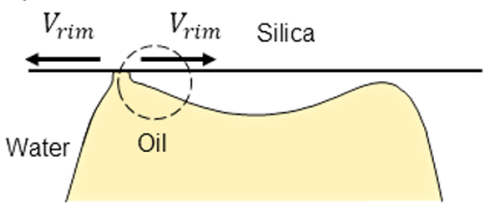

b)

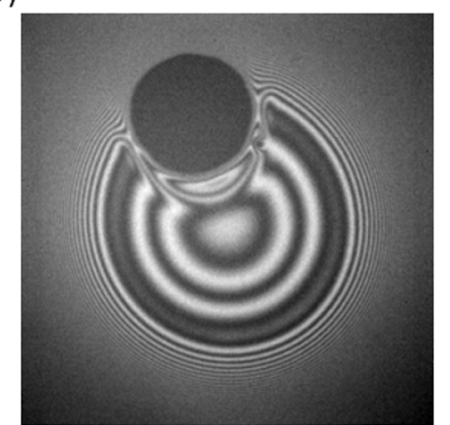

e)

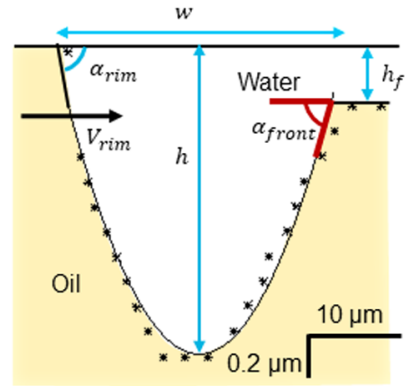

c)

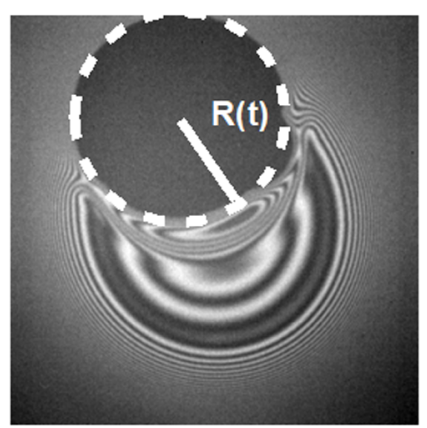

f)

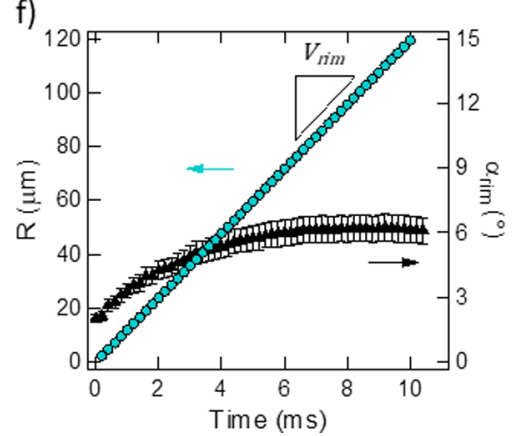

Figure 4. (a)-(c) Microscopy images: top views of the dewetting of water on silica within a water film trapped between oil and silica. [CTAB]/ $\mathrm{CMC}=10^{-3}$. Time interval between images: $2.8 \mathrm{~ms}$. Contact zones between oil and silica appear as black disks of radius $R$. Rings are equal thickness fringes. (d) Sketch of the oil/water interface when water recedes on silica against oil around an oil/silica contact zone. (e) Profile of the oil/water interface at the contact line from interferometry data (asterisks) evidencing a dewetting rim advancing on a trapped water film of thickness $h_{\mathrm{f}}=250 \mathrm{~nm}$. The line is a fit to a parabola. [CTAB] $/ \mathrm{CMC}=0$, time after nucleation $=17 \mathrm{~ms}$. The water wedge recedes with a velocity $V_{\text {rim }}$ and connects silica at an angle $\alpha_{\text {rim. }}$ (f) Triangle, rim contact angle $\alpha_{\text {rim }}$ vs time; circle, radius $R$ of the dewetted zone vs time. The slope determines $V_{\text {rim }}=12.1 \mathrm{~mm} \cdot \mathrm{s}^{-1},[\mathrm{CTAB}] / \mathrm{CMC}=10^{-3}$.

\section{RESULTS}

Dewetting Dynamics of Surfactant Solutions on Silica in Oil within a Trapped Water Film. First, for concentrations above $0.8 \mathrm{CMC}$, no water film puncture could be observed in our range of pressure value. For this reason, in this concentration range, shown in blue in Figure 5, no dynamic data was reported. At $5 \mathrm{CMC}$, the static receding contact angle $\alpha_{\mathrm{e}}$ is measured using a specific procedure: we initially approached an oil drop within pure water, which led to spontaneous dewetting of water on silica. Then, water was replaced in situ with a $5 \mathrm{CMC}$ solution and $\alpha_{\mathrm{e}}$ was then measured: it is found lower than at $0.8 \mathrm{CMC}$, but of large finite value $\left(40^{\circ}\right)$. In the past, no occurrence of dewetting was interpreted as total wetting condition for water on silica in oil, ${ }^{4,11}$ in apparent contradiction to the non-zero equilibrium contact angle we measure. Actually, zero occurrence of nucleation at $5 \mathrm{CMC}$ is instead attributed to a larger amplitude energetic barrier for nucleation due to confined and possibly structured surfactants of high concentration in the water film. $^{60,61}$ This will be the scope of the next article.

In the surfactant concentration range from 0 to $0.8 \mathrm{CMC}$, the aqueous film is observed to spontaneously puncture, and a rim always collects the dewetting aqueous solution, with an angle and velocity that are independent of time and squeezed water film thickness as soon as the dewetted zone is large enough. The rim dewetting angle and velocity are plotted as a function of the $\mathrm{CTAB}$ concentration in bulk in Figure 5. In addition, the edge of the dewetted area is observed to become all the more irregular that bulk concentration increases, as shown in Figure 6. As pointed out in the Introduction, dewetting of water in oil is driven by the gain in surface energy $\gamma\left(\cos \alpha_{\mathrm{e}}-\cos \alpha_{\mathrm{rim}}\right)$ between the equilibrium situation and dynamic one. We therefore systematically measured the equilibrium angle $\alpha_{\mathrm{e}}$ as the static receding contact angles at vanishing velocity at the edge of the drop (Figure 3c), after dewetting of water has occurred. Both angle values were averaged over two to six different experiments and are reported in Figure 5a. The equilibrium angle $\alpha_{\mathrm{e}}$ increases with bulk concentration from $40^{\circ}$ to $100^{\circ}$, while the dynamic rim contact angle slightly decreases between $6^{\circ}$ and $1^{\circ}$. Figure $5 \mathrm{~b}$ displays the surface energy contributions to the driving energy term corresponding to $\gamma \cos (\alpha)$ for the two sets of angles. A monotonic decrease of both surface energies with concentration is obtained, but more importantly, we find that the driving capillary term $\gamma\left(\cos \alpha_{\mathrm{e}}-\cos \alpha_{\text {rim }}\right)$ is roughly constant over the whole concentration range, in contrast with the contact line velocity displayed in Figure 5c: with no surfactant, it is close to $10 \mathrm{~mm} \cdot \mathrm{s}^{-1}$ and it abruptly decreases by three orders of magnitude as surfactant concentration increases between $10^{-2}$ and $0.8 \mathrm{CMC}$.

Dewetting Dynamics of Surfactant Solutions on Silica at the Edge of an Oil Drop. To understand this apparent contradiction, supplementary data on the wetting dynamics are needed to characterize the dissipative processes at stake in the vicinity of the contact line, as pointed out in Introduction. To this end, the large angle and low velocity range were explored by using side views of oil drops in contact with silica (Figure 3c). After spontaneous dewetting of the water film, oil was injected in or removed from the drop at rates varying between 0.1 and $300 \mu \mathrm{L} \cdot \mathrm{min}^{-1}$ and is depicted in Figure 7a. The contact angle $\alpha$ at the oil/water/silica contact line was measured as a function of its velocity $V$. Typical data 


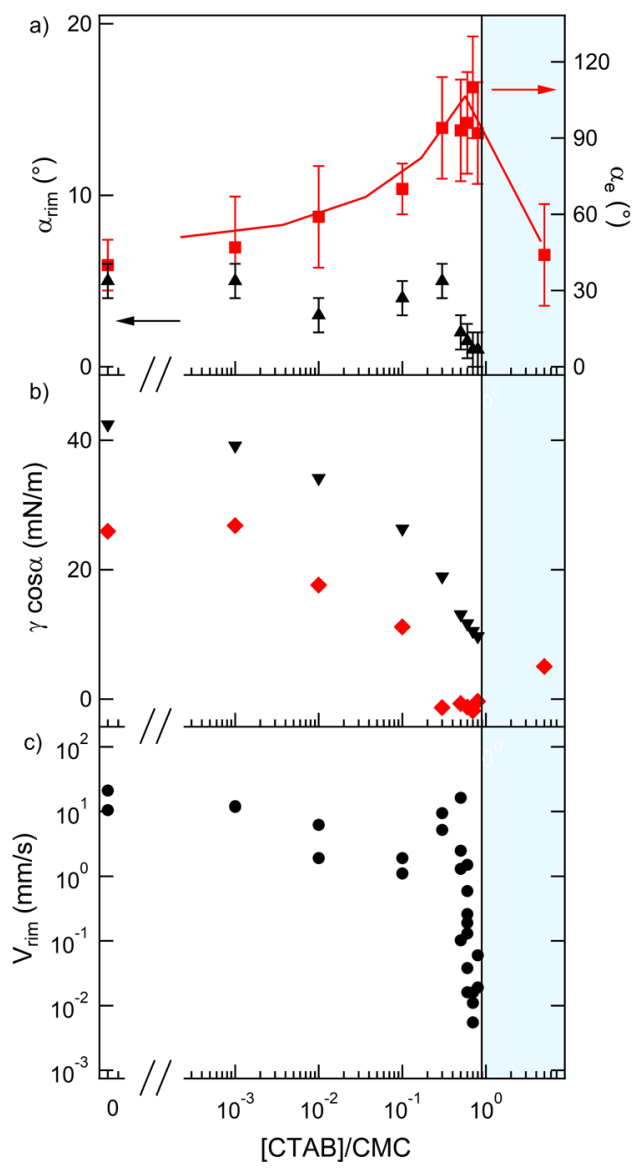

Figure 5. Dewetting dynamics at the triple line of the rim as a function of CTAB concentration (a) $\mathbf{\Delta}$, dynamic contact angle $\alpha_{\text {rim }}$; - (red), equilibrium contact angle $\alpha_{\mathrm{e}}$. (b) Surface energy contributions: $\nabla, \gamma \cos \alpha_{\text {rim }} ;$ (red), $\gamma \cos \alpha_{\mathrm{e}}$. Values are averages. (c) Contact line receding velocity measured at the edge of the rim. The blue zone corresponds to concentrations where the water film remains metastable at the level of pressure applied here.

are shown as an inset in Figure $7 \mathrm{~b}$ for both advancing and receding cases. The receding case is plotted in Figure $7 \mathrm{~b}$ for water and Figure 7c for CTAB solutions.

At all concentrations, in the small velocity range, the contact angle is independent of $V$ and equal to $\alpha_{\mathrm{e}}$. The plateau ends with a sharp decrease of the angle with velocity, at $V \sim 10^{-2}$ $\mathrm{m} / \mathrm{s}$ in pure water (Figure $7 \mathrm{~b}$ ), and at $V$-values, all the more low that more surfactants are added (Figure $7 \mathrm{c}$ ). The angle $\alpha_{\text {rim }}$ and velocity $V_{\text {rim }}$ of the rim are added to these graphs as stars. In all cases, the rim data nicely align with swollen drop data, demonstrating that both experiments explore the same physics. In the following, we offer to analyze the full contact line dynamics data to identify the relevant dissipative mechanisms, with and without surfactants. We will ultimately relate them to the adsorbed surfactant layers.

Dewetting Dynamics Driven by Friction at the Contact Line. Two contact lines dynamics models existing in literature are compared to the present data. In a purely hydrodynamic approach, the energy loss results from viscous dissipation in the corner formed by receding water in oil on silica, which yields the following relation between $\alpha$ and $V^{20,23,25,62}$

$$
\alpha_{\mathrm{e}}^{\beta}-\alpha^{\beta}=\mathrm{Ca} \ln \left(\frac{b}{a}\right) K
$$

$\alpha_{\mathrm{e}}$ is the receding equilibrium contact angle, $\mathrm{Ca}=\eta \mathrm{V} / \gamma$ is the capillary number with $\eta=0.89 \mathrm{mPa} \cdot \mathrm{s}$ the viscosity of the liquid wedge, $\gamma=41.9 \mathrm{mN} \cdot \mathrm{m}^{-1}$ is the interfacial tension between the two liquids, $a$ is the molecular-size length, and $b$ is the macroscopic scale that we can assume to be the typical size of the drop. This leads to a factor $\ln (b / a)$ of order 15 . The constants $K$ and $\beta$ depend on the viscosity ratio $\epsilon$ of the two liquid phases. Here, we have $\epsilon=\eta_{\mathrm{oil}} / \eta=1.4$. In the case of a viscosity ratio between the two fluids $\epsilon$ much smaller than 1 (e.g., a liquid drop in air) and $\alpha<3 \pi / 4$, eq 2 reduces to the equation derived by $\operatorname{Cox}^{19}$ and Voinov ${ }^{20}$ with $\beta=3$ and $K=9$. For $\epsilon=1.4$ (which is the ratio we have here for water/ dodecane), Fetzer et al. ${ }^{25}$ showed that Cox solution reduces to eq 2 , but with $\beta=2.52$ and $K=15.9$. We will use these values in the following. Here, the capillary number Ca varies between $10^{-5}$ and $10^{-3}$; therefore, it is always much smaller than unity. For pure water, eq 2 is plotted as a dash-dotted line on Figure $7 \mathrm{~b}$ with $\alpha_{\mathrm{e}}=40^{\circ}$. We observe that the capillary number at the vanishing contact angle is predicted at $\mathrm{Ca}=1.8 \cdot 10^{-2}(\mathrm{~V}=$ $\left.810^{-2} \mathrm{~m} \cdot \mathrm{s}^{-1}\right)$, while in the present experiments, this velocity is measured to be $V=10^{-2} \mathrm{~m} \cdot \mathrm{s}^{-1}$, which is one order of magnitude smaller than predicted by the hydrodynamic model. Thus, viscous dissipation plays a negligible role on the motion of the contact line, and the dissipation likely originates from a contact line friction of other, microscopic, origin.

In literature, ${ }^{18,22,24}$ dissipation at the contact line has alternatively been offered to originate from a friction-like mechanism at a molecular scale, located at the contact line. The contact line displacement is associated to molecular jumps between adsorption sites on the solid, which are thermally
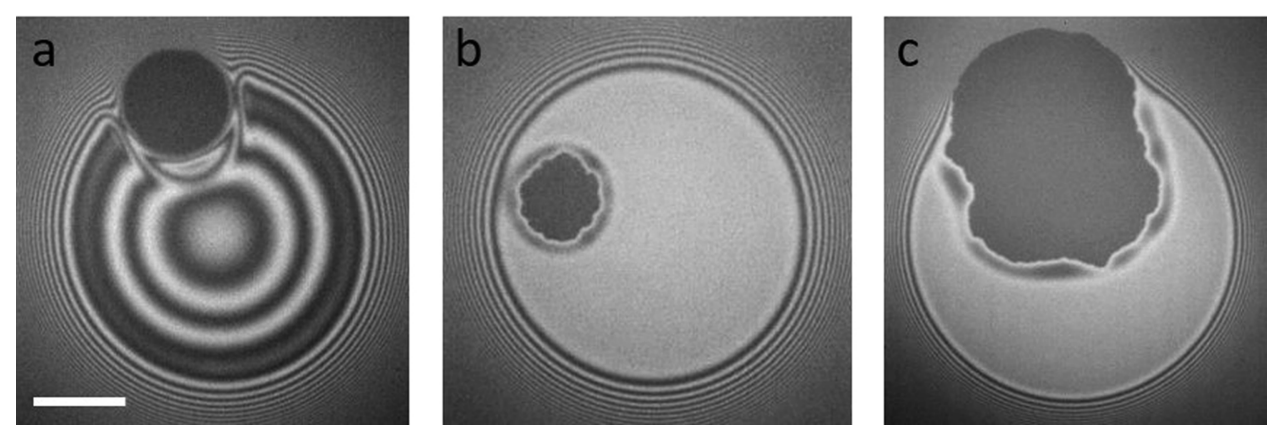

Figure 6. Microscopy images: top views of the dewetting of aqueous solutions of CTAB on silica within a film trapped between oil and silica. $[\mathrm{CTAB}] / \mathrm{CMC}=(\mathrm{a}) 10^{-3}$, (b) 0.5 , (c) 0.8 . The contact line is increasingly irregular as concentration increases. The white line represents $50 \mu \mathrm{m}$. 
(a)
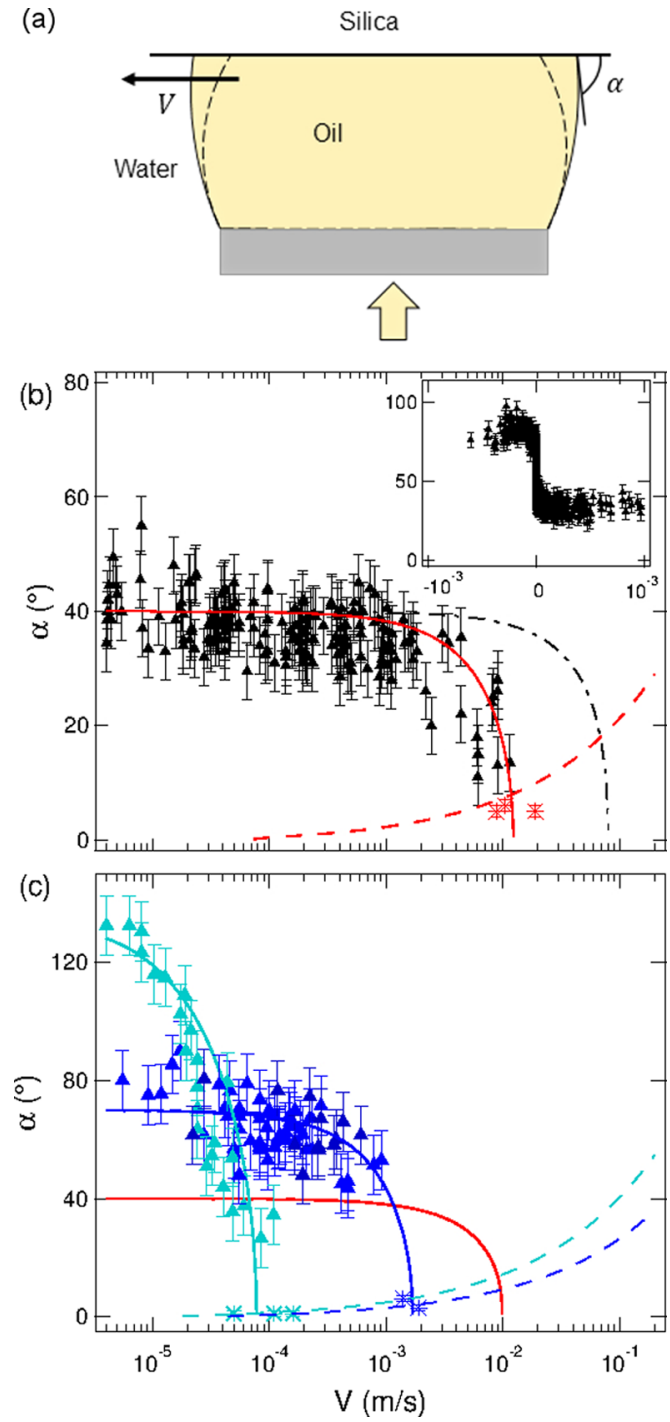

Figure 7. (a) Sketch of the swollen drop experiment: oil is injected to measure $V$ and $\alpha$ at the drop scale. (b, c) Dynamic receding contact angle $\alpha$ as a function of contact line velocity $V(\log$ scale $): \boldsymbol{\Lambda}$, swollen drop experiment; $*$, rim data. (b) Pure water, $\alpha_{\mathrm{e}}=40^{\circ}$ (c) (blue) $[\mathrm{CTAB}] / \mathrm{CMC}=0.1, \alpha_{\mathrm{e}}=70^{\circ}$ (cyan) $[\mathrm{CTAB}] / \mathrm{CMC}=0.7, \alpha_{\mathrm{e}}=$ $130^{\circ}$. (b) - -•-, Hydrodynamic model, eq 2 with $\beta=2.52, \mathrm{~K}=15.9$. $(\mathrm{b}, \mathrm{c})-$, Molecular kinetic model, eq4 with (red) $\zeta=0.8 \mathrm{~Pa} \cdot \mathrm{s}$; (blue) $\zeta=9.9 \mathrm{~Pa} \cdot \mathrm{s}$; (cyan) $\zeta=400 \mathrm{~Pa} \cdot \mathrm{s} .---$, hydrodynamic model eq 9 for the front angle of the rim (see Appendix) $\left(\beta=2.52, K=15.9, w / h_{\mathrm{f}}\right.$ $=100$, and value of oil/water interfacial tension $\gamma$ depends on CTAB concentration). (b) Inset: pure water: advancing and receding dynamic angle vs velocity $V$ (linear scale) from swollen/deswollen drop experiments.

activated, and the corresponding energy dissipation is denoted $F(V)$ so that the equation relating $\alpha$ and $V$ writes

$$
\cos \alpha=\cos \alpha_{\mathrm{e}}+\frac{F(V)}{\gamma}
$$

with $F(V)=2 n k_{\mathrm{B}} T \arcsin h\left(\frac{V}{2 K^{0} \lambda}\right)$, where $K^{0}$ is the jump frequency between two molecular sites, $\lambda$ is the distance between them, $n=1 / \lambda^{2}, k_{\mathrm{B}}$ is the Boltzmann's constant, and $T$ is the temperature. In the low $\mathrm{Ca}$ limit, eq 3 can be linearized ${ }^{24,31}$ using a molecular friction coefficient $\zeta=k_{\mathrm{B}} T /$ $K^{0} \lambda^{3}$. Eq 3 becomes
Eq 4 can be generalized to any friction mechanism whatever its physical or physico-chemical origin, provided that a friction coefficient $\zeta$ linearly relates the dissipative energy to velocity. ${ }^{29,30,63}$ Any kind of friction localized in the immediate vicinity of the contact line would lead to the same expression.

We have verified that in our case both eqs 3 and 4 give nearly the same values of $\zeta$ at all concentrations, demonstrating that friction dominates over viscous dissipation. Here, we first assume that the interfacial tension at the liquid interface can be taken as set by the bulk surfactant concentration. This hypothesis will be validated in the Discussion section. All data sets were successfully fitted to eq 4 with $\zeta$ as a fitting parameter: we find $\zeta \simeq 0.8 \mathrm{~Pa} \cdot \mathrm{s}$ for pure water, and $\zeta=10 \mathrm{~Pa} \cdot \mathrm{s}$ at $0.1 \mathrm{CMC}$, in agreement with values from literature on other but close systems: for dodecane in water on thiol-coated gold surfaces, Fetzer et al. ${ }^{25}$ found $\zeta$ values ranging from 1.6 to 3.7 $\mathrm{Pa} \cdot \mathrm{s}$; for a CTAB solution receding in air on either a polymer sealant substrate or polystyrene, Fell et al. ${ }^{1}$ found $\zeta$ values ranging from 4 to $6 \mathrm{~Pa} \cdot \mathrm{s}$. Figure 8 displays the variations of $\zeta$

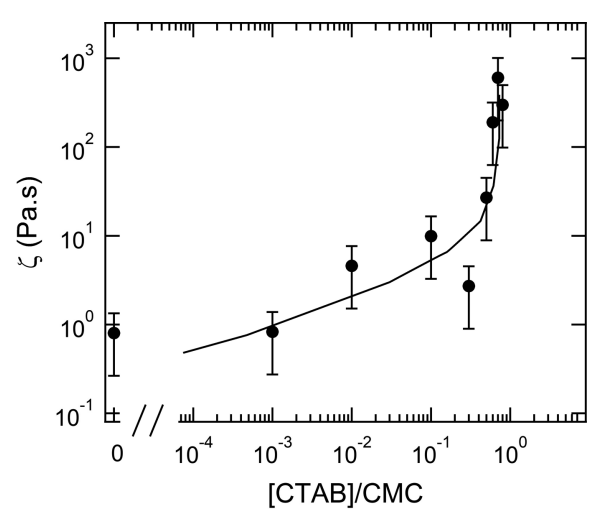

Figure 8. Friction coefficient $\zeta$ of the contact line receding on silica in $\mathrm{CTAB}$ solutions as a function of $\mathrm{CTAB}$ concentration normalized by CMC. The full line is a guide for the eyes.

with the $\mathrm{CTAB}$ concentration: $\zeta$ values were averaged over two to six experiments for each concentration. We find that the friction coefficient strongly increases with concentration, reaching almost $10^{3} \mathrm{~Pa} \cdot \mathrm{s}$ close to CMC.

In conclusion, we showed that, at all concentrations, the contact line dynamics is controlled by the balance between the gain of surface energy that acts as the driving force and a dissipation by friction at the contact line that cannot be reduced to hydrodynamics only. We further find that, although adding surfactants up to $0.8 \mathrm{CMC}$ seem to hydrophobize the silica/water interface as revealed by an increase in $\alpha_{\mathrm{e}}$, the dewetting of water from this hydrophobized interface is found to dramatically slow down because friction at the contact line considerably increases.

At this stage, a comment can be made on the shape of rim and the selection of its dewetting velocity, which is found independent of water film thickness. In Appendix, we solve this question in our very specific situation where friction at the contact line is several orders of magnitude larger than viscous dissipation. We show that the rims collecting the liquid are of constant curvature and geometrically constrained by both hydrodynamic dissipation and contact-line friction operating in parallel at the rear and front edges of the growing rim, as 
suggested by Bertrand et al. ${ }^{64}$ in a simpler one-liquid situation. As we shall see, our description is in very good agreement with our experimental results.

\section{DISCUSSION}

We now consider the different effects, involving surfactants, from which the dramatic slowing down of water receding dynamics within the squeezed water film could result. We first address the question of the magnitude of the interfacial surface tension at the contact line, especially if surfactant desorb from the solid surface as contact line recedes: surfactant desorption may lead to an increase of surfactant concentration, localized in the wedge. In literature on triple contact line dynamics with surfactants, ${ }^{1,2}$ the onset of a Marangoni flow at the liquid interface was discussed as a possible origin of the decrease of the contact angle with contact line velocity.

Surfactant-Induced Marangoni Effects at Oil/Water Interface Do Not Control the Dewetting Dynamics. The oil/water/silica triple line recedes and is depicted in Figure 4e. Note that the rim formed ahead of the contact line has a width over height aspect ratio of 100 . Therefore, from a hydrodynamic point of view, the situation amounts to the sketch of Figure 9. As the triple phase contact line moves, surfactants can either remain adsorbed on the silica surface or desorb, either fully or partially.

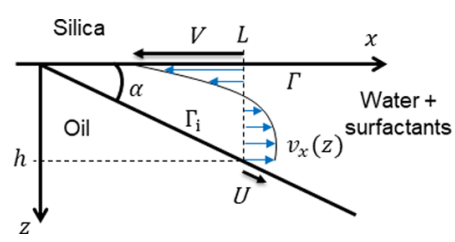

Figure 9. Sketch of the water wedge receding in oil on silica, in the reference frame of the contact line, with an angle $\alpha$ and a velocity $V$. $v_{x}(z)$ : water velocity profile in the lubrication approximation, $U$ : velocity at the water/oil interface, $\Gamma$ and $\Gamma_{i}$ : surfactant surface concentrations, respectively desorbed from the water/silica interface and adsorbed at the water/oil interface. $h$ and $L$ : characteristic length along the $z$-and $x$-axes, respectively (see text). For the sake of clarity, the surfactant molecules have not been represented.

That local increase can be opposed by three effects, namely, surfactant diffusion in the bulk, advection of the water solution in and out of the wedge $\left(v_{x}(z)\right)$, bulk diffusion, and interfacial advection at the oil/water interface $(U)$. The latter arises when gradients of surfactant concentration at the liquid interface generate a Marangoni flow. A detailed modelling of these transfers is given in the Supporting Information. The main results are summarized here. First, in the liquid wedge, the advection-diffusion of surfactants in the bulk is shown to be driven by diffusion only. Indeed, the Péclet number comparing surfactant transport by the flow and by diffusion writes: $\mathrm{Pe}=$ $\alpha h V / D$. Given the typical values of velocity, between $10^{-2}$ and $10^{-5} \mathrm{~m} \cdot \mathrm{s}^{-1}$, of the rim thickness $h$ of $400 \mathrm{~nm}$ and $D \sim 10^{-10}$ $\mathrm{m}^{2} \cdot \mathrm{s}^{-1}$, Pe remains smaller than 1 , which means that surfactant concentration $C$ obeys a diffusion equation: $\Delta C=0$. More precisely, transverse diffusion along $z$ (Figure 9) feeds the liquid interface with surfactants from a subsurface layer, which extends over the depletion length $\lambda$ : it is defined as the ratio $\Gamma_{i}^{\infty} / \mathrm{CMC}$ (where $\Gamma_{i}^{\infty}$ is the liquid interface concentration at saturation, see the Supporting Information). For CTAB, $\lambda \sim$ $2 \mu \mathrm{m}$, thus than larger than the rim height $h$. Hence, in the wedge, bulk concentration $C$ is homogeneous along the transverse direction, and the bulk solution of concentration $C(x)$ is in equilibrium with the liquid interface bearing a surface concentration $\Gamma_{i}(x)$. For CTAB, a Langmuir isotherm relates $C$ and $\Gamma_{i}$. In the following, we show that, in the wedge, the surfactants desorbed from the solid surface (flux $\Gamma V$ ) are mainly driven out of the wedge by a Marangoni flux at the liquid interface (flux $\Gamma_{i} U$ ), which is predominent over the diffusive transport along the longitudinal direction $x$. The diffusion equation, written now as a function of the distance $r$ to the contact line, is supplemented by two boundary conditions: no flux through the solid interface, and mass conservation of surfactants. The first condition imposes that $C$ decreases logarithmically with the distance $x$ to the contact line due to the wedge geometry. The second writes:

$$
\Gamma V=\Gamma_{i} U+D \alpha x \frac{\mathrm{d} C}{\mathrm{~d} x}
$$

where $U$ is the velocity at the liquid interface. It is set by the hydrodynamic boundary condition that imposes that the viscous tangential stress in water is balanced by the Marangoni stress arising from the gradient in surface tension along the interface. Note that the viscous stress in oil can be neglected because shear in oil develops over the typical length $R_{\mathrm{d}}$, the radius of the drop, which is orders of magnitude larger than $h=$ $\alpha x$. The liquid/liquid interface boundary condition therefore writes: $\eta \frac{U-V / 2}{h} \sim \frac{\mathrm{d} \gamma}{\mathrm{d} x}$, which allows to derive the Marangoni interfacial velocity $U$. The mass conservation eq 5 becomes:

$$
\Gamma V=\Gamma_{i} V / 2+\alpha x \frac{\mathrm{d} C}{\mathrm{~d} x}\left(D+\frac{\Gamma_{i}}{\eta} \frac{\mathrm{d} \gamma}{\mathrm{d} c}\right)
$$

where we use $\frac{\mathrm{d} \gamma}{\mathrm{d} x}=\frac{\mathrm{d} y}{\mathrm{dC}} \frac{\mathrm{dC}}{\mathrm{d} x}$. Eq 6 evidences longitudinal surfactant transfers result from two mechanisms, diffusion and Marangoni, that can be compared through the Marangoni number $\mathrm{Ma}=\frac{\Gamma_{i}}{\eta D} \frac{\mathrm{d} \gamma}{\mathrm{dC}}$. For CTAB, and close to CMC, $\Gamma_{i} \sim \Gamma_{i}^{\infty} \sim$ $2 \mu \mathrm{mol} \cdot \mathrm{m}^{-3}$ and the Gibbs equation yields: $\frac{\mathrm{d} \gamma}{\mathrm{dC}} \sim R T \Gamma_{i}^{\infty} / \mathrm{CMC} \sim 4 \cdot 10^{-3} \mathrm{Nm}^{2} \mathrm{~mol}^{-1}$. We find $\mathrm{Ma} \sim 10^{5}$ orders of magnitude larger than 1: surfactants desorbed from the solid interface are predominantly expelled from the wedge through the interfacial Marangoni flow. It follows that, at a distance $r$ from the contact line, the increase in concentration in the wedge relative to the solution concentration $C_{0}$ set at large distance $L$ can be written as

$$
\frac{\Delta C}{C_{0}} \sim \frac{1}{\alpha} \frac{V}{V_{\mathrm{c}}} \ln \frac{L}{r}
$$

where we assume $\Gamma$ and $\Gamma_{i}$ are of the same order of magnitude, and we introduce the capillary velocity $V_{c}=(\gamma / \eta)$. More importantly, the resulting interfacial tension decrease can also be calculated: $\Delta \gamma \sim \frac{\gamma}{\alpha} \frac{V}{V_{\mathrm{c}}} \ln \frac{L}{r}$.

In our conditions, $V_{\mathrm{c}}=0.6 \mathrm{~m} \cdot \mathrm{s}^{-1}$ is orders of magnitude larger than the velocities $V$ we measure. Consequently, even at a distance $r$ as close as $1 \mathrm{~nm}$ to the contact line, we find $\Delta \gamma \simeq$ $10^{-8} \mathrm{~N} \cdot \mathrm{m}^{-1}$ is negligible.

The Supporting Information details the full calculation of the concentration profiles in the wedge, by taking into account the part of the wedge where $C$ may become larger than CMC, and hence, no adsorption occurs at the liquid interface. It leads to the same conclusions: if surfactants desorb from the solid interface, they are expelled out of the wedge, and the oil/water 
interfacial tension is not modified and set by the bulk concentration in the tank. Finally, the onset of a Marangoni flow at the liquid-liquid interface changes the boundary condition for the flow in the wedge, but it only affects the viscous dissipation term in the contact line dynamics and by a numerical factor of order unity. In the present study, we found that friction at the contact line dominates over the viscous terms so that surfactant transfers are not expected to alter significantly either the driving capillary term or the dissipation term in eq 2.

Friction at the Contact Line Increases with Strongly Adsorbed Surfactants at the Silica Surface. In the following, we offer to discuss the variations of $\zeta$ and $\alpha_{\mathrm{e}}$ in relation to the amount of $C T A B$ adsorbed at the silica surface $\Gamma$, instead of bulk concentration. To do so, we first modify the adsorption isotherm of CTAB on silica (Figure 2) into a bulk concentration versus the surface concentration $\Gamma$ curve in Figure 10a. As detailed earlier, the value $\Gamma^{*} \sim 0.4 \mu \mathrm{mol} \cdot \mathrm{m}^{-2}$ discriminates the adsorption of strongly bound surfactants from the weakly bound ones. The variations of $\zeta$ as a function

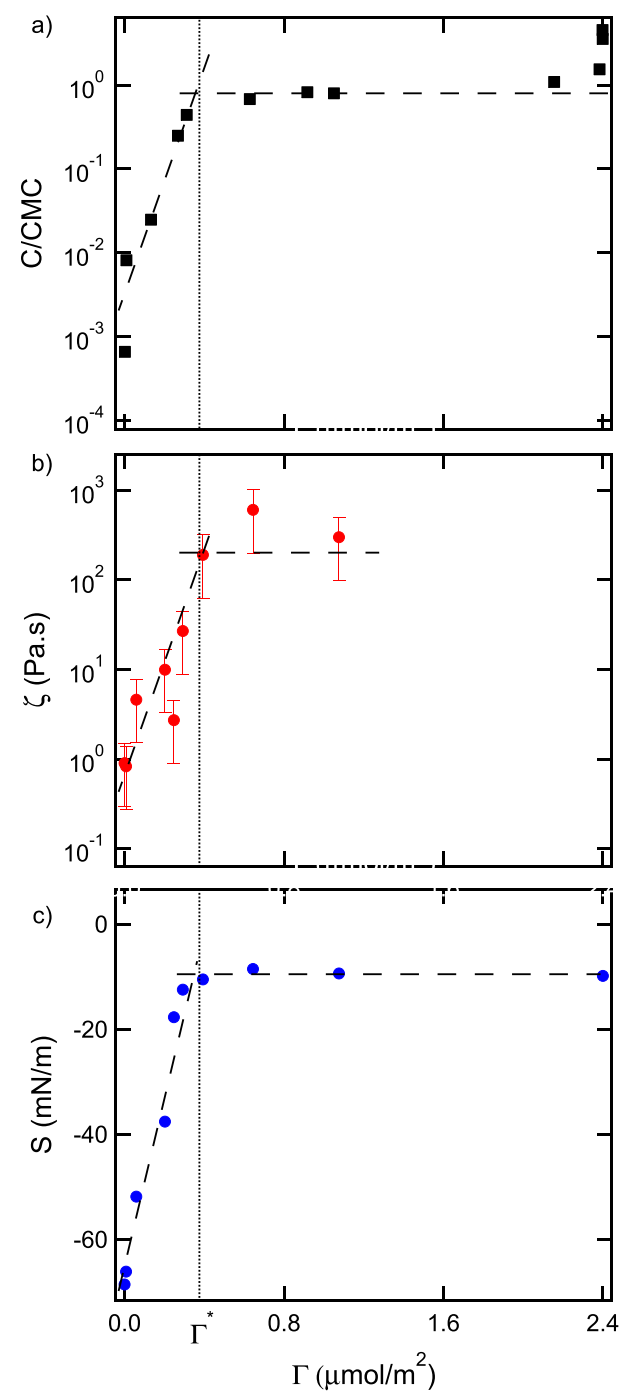

Figure 10. Variations with the CTAB surface concentration at water/ silica interface $\Gamma$ of (a) bulk surfactant concentration normalized by CMC, (b) friction coefficient $\zeta$, and (c) spreading parameter $S=-$ $\gamma\left(\cos \alpha_{\mathrm{e}}+1\right)$. Dashed lines: guides to the eyes evidencing an increase and a plateau above $\Gamma^{*} \sim 0.4 \mu \mathrm{mol} \cdot \mathrm{m}^{-2}$. of surface concentration on silica $\Gamma$ are plotted in Figure $10 \mathrm{~b}$. The coefficient $\zeta$ first increases with $\Gamma$ until it reaches a plateau above the threshold value $\Gamma^{*}$, which is remarkably the same as for the CTAB adsorption isotherm on silica.

On the other hand, following the path of previous studies, ${ }^{4,11}$ the affinity of silica for oil is characterized through the spreading parameter, denoted $S$, which compares the surface energies of silica wetted by water and of silica wetted by oil with water as a surrounding medium. A negative value of $S$ corresponds to oil partially wetting silica within water. Water receding is therefore all the more favored that $S$ is large. With our system and notations, $S=-\gamma\left(\cos \alpha_{\mathrm{e}}+1\right)$ can be computed from the measured interfacial tension and equilibrium angles. The results are plotted in Figure $10 \mathrm{c}$ as a function of surface concentration: $S$ first increases with $\Gamma$, then also saturates at the same remarkable value $\Gamma^{*}$. Below $\Gamma^{*}$, first, the increase of $S$ means that hydrophobicity of silica increases with the amount of surfactants adsorbed on the solid surface, in good agreement with a picture where $\mathrm{CTAB}$ tails are exposed to the liquids. In contrast, in the same $\Gamma$ range, the friction coefficient increases, indicating that receding of water becomes slower as surface hydrophobicity is enhanced: no direct link is observed between the spreading parameter and friction coefficient in our case. Our observations are also at variance with results from literature where dynamic wetting of solids by liquids was analyzed using the adhesion energy defined as $S+2 \gamma:{ }^{22,24}$ the friction coefficient was found to increase exponentially with adhesion energy. ${ }^{27}$ In the present experiments, friction is found to decrease with adhesion energy. Clearly, in the present system, that picture is not adequate either.

Instead, combining our results on adsorption at the silica surface (Figure 10a), friction (Figure 10b), and spreading parameter (Figure 10c) allows us to offer the following picture. Both $S$ and $\zeta$ increase with $\Gamma$ below $\Gamma^{*}$, that is, when only strongly adsorbed surfactants are increasingly covering the silica surface. Then, both $S$ and $\zeta$ saturate to a plateau value above $\Gamma^{*}$, namely, when any adsorbed additional surfactant is bound to the surface through low energy interactions. Note that the $S$-value measured at $\Gamma^{\infty}=2.4 \mu \mathrm{mol} \cdot \mathrm{m}^{2}$ where the surfactant forms a complete bilayer also belongs to this plateau. In other words, the strongly bound surfactants, of concentration $\Gamma<\Gamma^{*}$, do contribute to both the oil/silica interactions and the friction at the contact line. In contrast, for $\Gamma>\Gamma^{*}$, weakly bound surfactants, therefore at a surface concentration equal to $\Gamma-\Gamma^{*}$, neither contribute to oil/silica interactions nor to friction at the contact line, which are both set by the strongly bound surfactant at a concentration $\Gamma^{*}$. This suggests that, as water recedes on the adsorbed layer of $\mathrm{CTAB}$, the strongly bound CTABs are trapped between oil and silica and do not desorb, while the weakly bound CTABs are desorbed and removed from the wedge by a Marangoni flow at the oil/ water interface. We conclude that both the kinetics and thermodynamics of dewetting ignore the weakly adsorbed surfactants that desorb from silica. Conversely, surfactants that are adsorbed through strong electrostatic interactions change the silica surface from hydrophilic to hydrophobic, but this enhanced affinity for oil that should accelerate water dewetting plays a negligible role. Instead, strongly bound surfactants impair the dewetting dynamics through a strong increase of the contact line friction. By combining our observations that contact lines becomes all the more irregular that concentration increases in Figure 6, with the description from literature of the 
patterns progressively formed by adsorbed CTAB (see Experimental Section), we offer to ascribe the contact line friction increase to the growth of surface heterogeneities: as the surface concentration of strongly bound surfactants increase, monolayered-like patches of surfactants progressively form, grow, and connect, and the silica surface exhibits more complex patterns on which the contact line gets pinned.

\section{CONCLUSIONS}

The receding dynamics of water on silica in oil is shown to be controlled by the gain in surface energy, which is dissipated through friction at the contact line. Upon the addition of cationic surfactants in the water phase, up to approximately 0.8 $\mathrm{CMC}$, water spontaneously dewets, and the affinity of oil for silica is found to increase while the dewetting dynamics of water is heavily impaired: it can be described by a huge increase of the friction coefficient at the contact line. We show that these effects are fully described by the quantity of surfactants adsorbed at the silica surface and their transfers. We show that: (i) Only electrostatically bound surfactants at the silica surface contribute to friction at the contact line, through contact line pinning on surfactants patterns. ${ }^{51}$ (ii) Surfactant bound to silica through weaker interactions desorbs as the contact line recedes, without contributing to friction. (iii) The gain in affinity for oil of surfactant-covered silica has much less positive impact on the water dewetting dynamics than does heterogeneity-induced contact friction in a negative way. From a practical point of view, our results show that cationic surfactants efficiently control the wetting of oil drops on silica in water up to a threshold concentration above which additional surfactants desorb and no longer contribute. In the present case, threshold concentration values are $\Gamma=0.4$ $\mu \mathrm{mol} \cdot \mathrm{L}^{-1}$ and $[\mathrm{CTAB}] \sim 0.5 \mathrm{CMC}$. We believe that such systems could be interestingly used to relate the empirical macroscopic friction coefficient to the patterns formed by adsorbed surfactants and elucidate its origin. Finally, we find that, in our experimental conditions, at $\mathrm{CTAB}$ concentrations larger than $0.8 \mathrm{CMC}$, the water film squeezed between oil and silica is never found to puncture. These cases will be detailed in a forthcoming paper, but still, we find altogether that CTAB added to the water delays the formation of oil wet silica zones by either dramatically slowing down the water dewetting dynamics or preventing their nucleation.

\section{APPENDIX A}

In the present state of literature, no available theory describes the dewetting velocity selection in the present case, namely, two liquids on a solid with triple line friction. The sole available references hold, in principle, for a single liquid in air. $^{59,64,65}$ We build here a specific model that is not far from the one offered by Bertrand et al., ${ }^{64}$ at least in the limit of large molecular over viscous dissipation ratio $\zeta / \eta$. In the present experiments, this ratio varies between $10^{3}$ and $10^{6}$ and is indeed large. The physical ingredients are as follows: (i) the oil/water/solid triple line recedes as described above, with a dynamics controlled by the difference between dynamic contact angle and equilibrium contact angle and a friction mechanism at the triple contact line: the dynamics at the rear follows eq 4. On the other hand, the rim advances on a water film so that (ii) the "equilibrium" contact angle of the rim on the film is zero, while the only relevant dissipative mechanism is viscous: the dynamics at the advancing front is described by eq 2 with $\alpha_{\mathrm{e}}=0$. Here, we assume that the two-liquid model holds, and we take $K=-15.9, \beta=2.52$. Furthermore, inspired by available works on "effective" contact lines sliding on a liquid film or prewetted surface, ${ }^{65-68}$ we assume that the logarythmic term $\ln (b / a)$ is approximately equal to $\ln \left(\mathrm{Ca}^{1 / 3} w /\right.$ $h_{\mathrm{f}}$ ), where $\mathrm{Ca}=\eta V / \gamma$ is the capillary number, $w$ is the rim width, and $h_{\mathrm{f}}$ is the water film thickness. Following the path of Redon et al., ${ }^{59}$ the hypothesis is further made that (iii) the front and rear velocities of the rim are equal. In the present experiment, this hypothesis holds as soon as the hole is large enough (typically $40 \mu \mathrm{m}$ in Figure 4f). (iv) Finally, the shape of the rim is assumed to be a spherical cap of uniform curvature set by Laplace pressure. ${ }^{59,64}$ From this, the triple line contact angle $\alpha$ and front angle connecting the rim to the film $\alpha_{\text {front }}$ are equal provided that the film thickness $h_{\mathrm{f}}$ is negligible compared to the rim height $h$.

As shown in Figure $4 \mathrm{f}$, the front angle can be measured at the inflexion point where the rim connects to the film: we find $\alpha_{\text {front }}=4^{\circ} \pm 1$ while $\alpha=6^{\circ}$. The data are reported in Figure 11

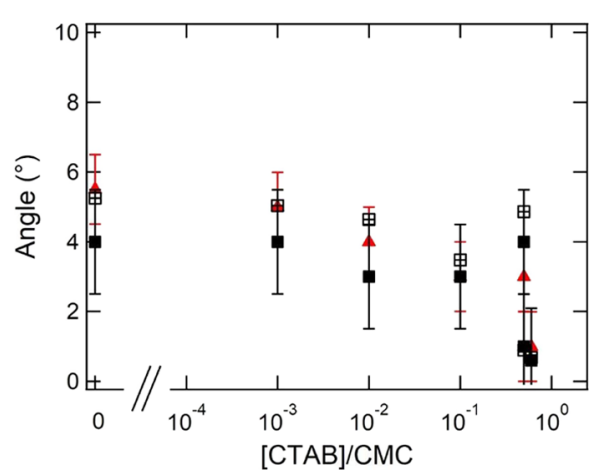

Figure 11. $\Delta$ (red), contact angle $\alpha_{\text {rim }}$ measured at the rear of the rim as a function of CTAB concentration; $\mathbf{\square}$, measured front angle $\alpha_{\text {front }}$ between the rim and the film; $\square$, angle solution to eq 9 using measured values of $V, w / h_{\mathrm{f}}, \gamma, \eta=0.9 \mathrm{mPa} \cdot \mathrm{s}, K=-15.9$, and $\beta=2.52$

for all CTAB concentrations. Note that the front angle is measured with high uncertainty due to the bell shape of the dimple. Nevertheless, the front and rear angles are found almost equal within experimental uncertainty, as soon as the rear and front velocities are measured equal, and in all the concentration range tested. Hence, hypotheses (iii) and (iv) are experimentally verified. It follows that the rim velocity $V$ should obey both eqs 4 and 2 with $\alpha \simeq \alpha_{\text {front }}$

$$
\begin{aligned}
& \cos \alpha=\cos \alpha_{\mathrm{e}}+\frac{\zeta V}{\gamma} \\
& \alpha^{\beta}=K \frac{\eta V}{\gamma} \ln \left[\left(\frac{\eta V}{\gamma}\right)^{1 / 3} \frac{w}{h_{\mathrm{f}}}\right]
\end{aligned}
$$

For equilibrium contact angles $\alpha_{\mathrm{e}}$ smaller than $50^{\circ}$, eq 8 can be linearized and $V$ obeys the following implicite equation

$$
V=\frac{\gamma}{\eta K \ln \left[\left(\frac{\eta V}{\gamma}\right)^{1 / 3} \frac{w}{h_{\mathrm{f}}}\right]}\left(\alpha_{\mathrm{e}}^{2}-2 \frac{\zeta V}{\gamma}\right)^{\beta / 2}
$$

Eq 10 was solved for each $\mathrm{CTAB}$ concentration, using measured values of the width of the rim $w$ and the film height $h_{\mathrm{f}}$. Over the whole range of concentration tested, $w$ over $h_{\mathrm{f}}$ 


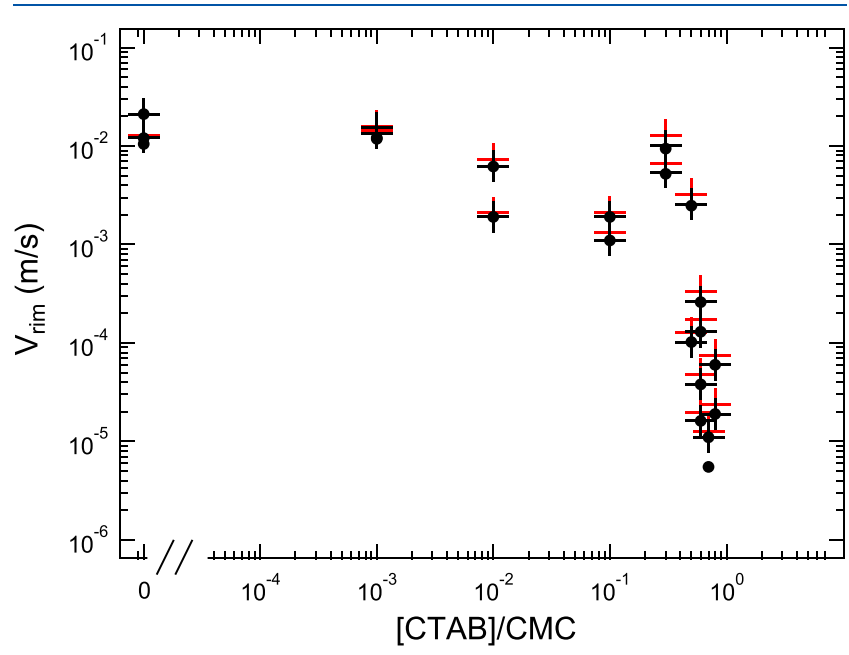

Figure 12. 0 , contact line receding velocity at the rear of the rim as a function of CTAB concentration; +, solution to eq $10 ;+$ (red), solution to combined eqs 8 and 9. Both resolutions were based on measured values of $w / h_{\mathfrak{f}} \zeta, \gamma, \alpha_{\mathrm{e}} ; \eta=0.9 \mathrm{mPa} \cdot \mathrm{s}, K=-15.9$, and $\beta=$ 2.52

당 orcid.org/0000-0002-7829-0795;

Email: emilie.verneuil@espci.fr

\section{Authors}

Gaëlle Rondepierre - Soft Matter Sciences and Engineering (SIMM), ESPCI Paris, PSL University, Sorbonne Université,
CNRS UMR 7615, Paris F-75005, France; TOTAL SA, Pôle Etudes et Recherche de Lacq, Lacq F-64170, France; Laboratoire Physico-chimie des Interfaces Complexes, ESPCI Paris, Paris F-75231, France

Franz De Soete - Soft Matter Sciences and Engineering (SIMM), ESPCI Paris, PSL University, Sorbonne Université, CNRS UMR 7615, Paris F-75005, France; TOTAL SA, Pôle Etudes et Recherche de Lacq, Lacq F-64170, France; Laboratoire Physico-chimie des Interfaces Complexes, ESPCI Paris, Paris F-75231, France

Nicolas Passade-Boupat - TOTAL SA, Pôle Etudes et Recherche de Lacq, Lacq F-64170, France; Laboratoire Physico-Chimie des Interfaces Complexes, CHEMSTARTUP, Lacq F-64170, France; 이이.org/0000-0003-2913-0864

François Lequeux - Soft Matter Sciences and Engineering (SIMM), ESPCI Paris, PSL University, Sorbonne Université, CNRS UMR 7615, Paris F-75005, France; Laboratoire Physico-chimie des Interfaces Complexes, ESPCI Paris, Paris F-75231, France; (1) orcid.org/0000-0003-4076-3988

Laurence Talini - Soft Matter Sciences and Engineering (SIMM), ESPCI Paris, PSL University, Sorbonne Université, CNRS UMR 7615, Paris F-75005, France; Laboratoire Physico-chimie des Interfaces Complexes, ESPCI Paris, Paris F-75231, France

Laurent Limat - Laboratoire Matière et Systèmes Complexes, Université de Paris, CNRS UMR 7057, Paris F-75013, France

Complete contact information is available at:

https://pubs.acs.org/10.1021/acs.langmuir.0c02746

\section{Notes}

The authors declare no competing financial interest.

\section{ACKNOWLEDGMENTS}

This work benefited from meetings within the French working group GDR CNRS 2019 "Solliciter LA Matière Molle" (SLAMM).

\section{REFERENCES}

(1) Fell, D.; Auernhammer, G.; Bonaccurso, E.; Liu, C.; Sokuler, R.; Butt, H.-J. Influence of Surfactant Concentration and Background Salt on Forced Dynamic Wetting and Dewetting. Langmuir 2011, 27, 2112-2117.

(2) Fell, D.; Pawanrat, N.; Bonaccurso, E.; Butt, H.-J.; Auernhammer, G. K. Influence of Surfactant Transport Suppression on Dynamic Contact Angle Hysteresis. Colloid Polym. Sci. 2013, 291, 361-366.

(3) Henrich, F.; Fell, D.; Truszkowska, D.; Weirich, M.; Anyfantakis, M.; Nguyen, T.-H.; Wagner, M.; Auernhammer, G. K.; Butt, H.-J. Influence of surfactants in forced dynamic dewetting. Soft Matter 2016, 12, 7782-7791.

(4) Churaev, N. V.; Ershov, A. P.; Esipova, N. E.; Iskandarjan, G. A.; Madjarova, E. A.; Sergeeva, I. P.; Sobolev, V. D.; Svitova, T. F.; Zakharova, M. A.; Zorin, Z. M.; Poirier, J.-E. Interaction of Oil Droplets with Silica Surfaces. Colloids Surf., A 1994, 91, 97-112.

(5) Stoebe, T.; Hill, R. M.; Ward, M. D.; Davis, H. T. Enhanced Spreading of Aqueous Films Containing Ionic Surfactants on Solid Substrates. Langmuir 1997, 13, 7276-7281.

(6) Starov, V. M.; Kosvintsev, S. R.; Velarde, M. G. Spreading of Surfactant Solutions over Hydrophobic Substrates. J. Colloid Interface Sci. 2000, 227, 185-190.

(7) Cachile, M.; Cazabat, A. M. Spontaneous Spreading of Surfactant Solutions on Hydrophilic Surfaces: $\mathrm{C}_{n} \mathrm{E}_{m}$ in Ethylene and Diethylene Glycol. Langmuir 1999, 15, 1515-1521. 
(8) Chaudhuri, R. G.; Paria, S. Dynamic Contact Angles on PTFE Surface by Aqueous Surfactant Solution in the Absence and Presence of Electrolytes. J. Colloid Interface Sci. 2009, 337, 555-562.

(9) Thiele, U.; Snoeijer, J. H.; Trinschek, S.; John, K. Equilibrium Contact Angle and Adsorption Layer Properties with Surfactants. Langmuir 2018, 34, 7210-7221.

(10) Serrano-Saldaña, E.; Domínguez-Ortiz, A.; Pérez-Aguilar, H.; Kornhauser-Strauss, I.; Rojas-González, F. Wettability of Solid/Brine/ n-Dodecane Systems: Experimental Study of the Effects of Ionic Strength and Surfactant Concentration. Colloids Surf., A 2004, 241, 343-349.

(11) Waghmare, P. R.; Das, S.; Mitra, S. K. Under-Water Superoleophobic Glass: Unexplored Role of the Surfactant-Rich Solvent. Sci. Rep. 2013, 3, 1862.

(12) Haidara, H.; Vonna, L.; Schultz, J. Surfactant-Induced Wetting Singularities in Confined Solid-Liquid-Liquid Systems: Kinetic and Dynamic Aspects. J. Adhes. Sci. Technol. 1999, 13, 1393-1403.

(13) Stammitti-Scarpone, A.; Acosta, E. J. Solid-Liquid-Liquid Wettability of Surfactant-Oil-Water Systems and Its Prediction around the Phase Inversion Point. Langmuir 2019, 35, 4305-4318.

(14) de Gennes, P. G. Wetting: Statics and Dynamics. Rev. Mod. Phys. 1985, 57, 827-863.

(15) Churaev, N. V. Wetting Films and Wetting. Rev. Phys. Appl. 1988, 23, 975-987.

(16) Le Grand, N.; Daerr, A.; Limat, L. Shape and Motion of Drops Sliding down an Inclined Plane. J. Fluid Mech. 2005, 541, 293.

(17) Snoeijer, J. H.; Andreotti, B. Moving Contact Lines: Scales, Regimes, and Dynamical Transitions. Annu. Rev. Fluid Mech. 2013, 45, 269-292.

(18) Blake, T. D.; Haynes, J. M. Kinetics of Liquid-Liquid Displacement. J. Colloid Interface Sci. 1969, 30, 421-423.

(19) Cox, R. G. The Dynamics of the Spreading of Liquids on a Solid Surface. Part 2. Surfactants. J. Fluid Mech. 1986, 168, 195.

(20) Voinov, O. V. Hydrodynamics of Wetting. Fluid Dyn. 1977, 11, 714-721.

(21) Petrov, P.; Petrov, I. A Combined Molecular-Hydrodynamic Approach to Wetting Kinetics. Langmuir 1992, 8, 1762-1767.

(22) Goossens, S.; Seveno, D.; Rioboo, R.; Vaillant, A.; Conti, J.; De Coninck, J. Can We Predict the Spreading of a Two-Liquid System from the Spreading of the Corresponding Liquid-Air Systems? Langmuir 2011, 27, 9866-9872.

(23) de Gennes, P.-G.; Brochard-Wyart, F.; Quere, D. Capillarity and Wetting Phenomena: Drops, Bubbles, Pearls, Waves; SpringerVerlag: New York, 2004.

(24) Seveno, D.; Blake, T. D.; Goossens, S.; De Coninck, J. Predicting the Wetting Dynamics of a Two-Liquid System. Langmuir 2011, 27, 14958-14967.

(25) Fetzer, R.; Ramiasa, M.; Ralston, J. Dynamics of Liquid-Liquid Displacement. Langmuir 2009, 25, 8069-8074.

(26) Ramiasa, M.; Ralston, J.; Fetzer, R.; Sedev, R. Contact Line Friction in Liquid-Liquid Displacement on Hydrophobic Surfaces. The Journal of Physical Chemistry C 2011, 115, 24975-24986.

(27) Duvivier, D.; Seveno, D.; Rioboo, R.; Blake, T. D.; De Coninck, J. Experimental Evidence of the Role of Viscosity in the Molecular Kinetic Theory of Dynamic Wetting. Langmuir 2011, 27, 1301513021.

(28) Rolley, E.; Guthmann, C. Dynamics and Hysteresis of the Contact Line between Liquid Hydrogen and Cesium Substrates. Phys. Rev. Lett. 2007, 98, 166105.

(29) Carlson, A.; Bellani, G.; Amberg, G. Universality in Dynamic Wetting Dominated by Contact-Line Friction. Physical Review E 2012, 85, No. 045302.

(30) Wang, Y.; Amberg, G.; Carlson, A. Local Dissipation Limits the Dynamics of Impacting Droplets on Smooth and Rough Substrates. Physical Review Fluids 2017, 2, No. 033602.

(31) Primkulov, B. K.; Lin, F.; Xu, Z. Microscale Liquid-Liquid Displacement Dynamics: Molecular Kinetic or Hydrodynamic Control. Colloids Surf., A 2016, 497, 336-343.
(32) de Ruijter, M. J.; Blake, T. D.; De Coninck, J. Dynamic Wetting Studied by Molecular Modeling Simulations of Droplet Spreading. Langmuir 1999, 15, 7836-7847.

(33) Hoffman, R. L. A Study of the Advancing Interface. J. Colloid Interface Sci. 1975, 50, 14.

(34) Karapetsas, G.; Craster, R. V.; Matar, O. K. On SurfactantEnhanced Spreading and Superspreading of Liquid Drops on Solid Surfaces. J. Fluid Mech. 2011, 670, 5-37.

(35) Kabalnov, A. Monolayer Frustration Contributions to Surface and Interfacial Tensions: Explanation of Surfactant Superspreading. Langmuir 2000, 16, 2595-2603.

(36) Wang, X.; Venzmer, J.; Bonaccurso, E. Surfactant-Enhanced Spreading of Sessile Water Drops on Polypropylene Surfaces. Langmuir 2016, 32, 8322-8328.

(37) Chan, K. Y.; Borhan, A. Surfactant-Assisted Spreading of a Liquid Drop on a Smooth Solid Surface. J. Colloid Interface Sci. 2005, 287, 233-248.

(38) Asatekin, A.; Mayes, A. M. Oil Industry Wastewater Treatment with Fouling Resistant Membranes Containing Amphiphilic Comb Copolymers. Environ. Sci. Technol. 2009, 43, 4487-4492.

(39) Zhu, X.; Dudchenko, A.; Gu, X.; Jassby, D. SurfactantStabilized Oil Separation from Water Using Ultrafiltration and Nanofiltration. J. Membr. Sci. 2017, 529, 159-169.

(40) Wang, Z.; Hou, D.; Lin, S. Composite Membrane with Underwater-Oleophobic Surface for Anti-Oil-Fouling Membrane Distillation. Environ. Sci. Technol. 2016, 50, 3866-3874.

(41) Rosen, M. J.; Kunjappu, J. T. Surfactants and Interfacial Phenomena; John Wiley \& Sons, Ltd, 2012; pp. 39-201.

(42) Fainerman, V. B.; Aksenenko, E. V.; Mucic, N.; Javadi, A.; Miller, R. Thermodynamics of Adsorption of Ionic Surfactants at Water/Alkane Interfaces. Soft Matter 2014, 10, 6873-6887.

(43) Moulik, S. P.; Haque, M. E.; Jana, P. K.; Das, A. R. Micellar Properties of Cationic Surfactants in Pure and Mixed States. J. Phys. Chem. 1996, 100, 701-708.

(44) Zdziennicka, A.; Szymczyk, K.; Krawczyk, J.; Jańczuk, B. Activity and Thermodynamic Parameters of Some Surfactants Adsorption at the Water-Air Interface. Fluid Phase Equilib. 2012, $318,25-33$.

(45) Kedar, V.; Bhagwat, S. S. Effect of Salinity on the IFT between Aqueous Surfactant Solution and Crude Oil. Pet. Sci. Technol. 2018, $36,835-842$.

(46) Velegol, S. B.; Fleming, B. D.; Biggs, S.; Wanless, E. J.; Tilton, R. D. Counterion Effects on Hexadecyltrimethylammonium Surfactant Adsorption and Self-Assembly on Silica. Langmuir 2000, 16, 2548-2556.

(47) Paria, S.; Khilar, K. C. A Review on Experimental Studies of Surfactant Adsorption at the Hydrophilic Solid-Water Interface. Adv. Colloid Interface Sci. 2004, 110, 75-95.

(48) Atkin, R.; Craig, V. S. J.; Biggs, S. Adsorption Kinetics and Structural Arrangements of Cationic Surfactants on Silica Surfaces. Langmuir 2000, 16, 9374-9380.

(49) Cases, J.-M.; Mielczarski, J.; Mielczarska, E.; Michot, L. J.; Villiéras, F.; Thomas, F. Ionic Surfactants Adsorption on Heterogeneous Surfaces. C. R. Geosci. 2002, 14.

(50) Theodoly, O.; Cascão-Pereira, L.; Bergeron, V.; Radke, C. J. A Combined Streaming-Potential Optical Reflectometer for Studying Adsorption at the Water/Solid Surface ${ }^{\dagger}$. Langmuir 2005, 21, 1012710139.

(51) Kékicheff, P.; Contal, C. Cationic-Surfactant-Coated Mica Surfaces below the Critical Micellar Concentration: 1. Patchy Structures As Revealed by Peak Force Tapping AFM Mode. Langmuir 2019, 35, 3087-3107.

(52) Atkin, R.; Craig, V. S. J.; Wanless, E. J.; Biggs, S. Mechanism of Cationic Surfactant Adsorption at the Solid-Aqueous Interface. Adv. Colloid Interface Sci. 2003, 103, 219-304.

(53) Zhu, B.-Y.; Gu, T.; Zhao, X. General Isotherm Equation for Adsorption of Surfactants at Solid/Liquid Interfaces. Part 2. Applications. J. Chem. Soc., Faraday Trans. 1 1989, 85, 3819. 
(54) Bluteau, L.; Bourrel, M.; Passade-Boupat, N.; Talini, L.; Verneuil, E.; Lequeux, F. Water Film Squeezed between Oil and Solid: Drainage towards Stabilization by Disjoining Pressure. Soft Matter 2017, 13, 1384-1395.

(55) Gee, M. L.; Goodall, D. G.; Stevens, G. W. Imaging of Droplet Profiles During Drainage of a Film Between a Droplet and an Interface. Oil \& Gas Science and Technology 2001, 56, 33-40.

(56) Lockie, H.; McLean, S.; Dagastine, R. R. Hindered Diffusion of an Oil Drop Under Confinement and Surface Forces. The Journal of Physical Chemistry Letters 2011, 2, 2472-2477.

(57) Zhang, X.; Manica, R.; Tang, Y.; Tchoukov, P.; Liu, Q.; Xu, Z. Probing Boundary Conditions at Hydrophobic Solid-Water Interfaces by Dynamic Film Drainage Measurement. Langmuir 2018, 34, 12025-12035.

(58) Bazazi, P.; Sanati-Nezhad, A.; Hejazi, S. H. Wetting Dynamics in Two-Liquid Systems: Effect of the Surrounding Phase Viscosity. Physical Review E 2018, 97, No. 063104.

(59) Redon, C.; Brochard-Wyart, F.; Rondelez, F. Dynamics of Dewetting. Phys. Rev. Lett. 1991, 66, 715-718.

(60) Danov, K. D.; Basheva, E. S.; Kralchevsky, P. A.; Ananthapadmanabhan, K. P.; Lips, A. The Metastable States of Foam Films Containing Electrically Charged Micelles or Particles: Experiment and Quantitative Interpretation. Adv. Colloid Interface Sci. 2011, 168, 50-70.

(61) Bergeron, V.; Radke, C. J. Equilibrium Measurements of Oscillatory Disjoining Pressures in Aqueous Foam Films. Langmuir 1992, 8, 3020-3026.

(62) Cox, R. G. The Dynamics of the Spreading of Liquids on a Solid Surface. Part 1. Viscous Flow. J. Fluid Mech. 1986, 168, 169.

(63) Wang, J.; Do-Quang, M.; Cannon, J. J.; Yue, F.; Suzuki, Y.; Amberg, G.; Shiomi, J. Surface Structure Determines Dynamic Wetting. Sci. Rep. 2015, 5, 8474.

(64) Bertrand, E.; Blake, T. D.; De Coninck, J. Dynamics of Dewetting. Colloids Surf., A 2010, 369, 141-147.

(65) Snoeijer, J. H.; Eggers, J. Asymptotic Analysis of the Dewetting Rim. Physical Review E 2010, 82, No. 056314.

(66) Voinov, O. V. Inclination angles of the boundary in moving liquid layers. Journal of Applied Mechanics and Technical Physics 1977, $18,216-222$.

(67) Chen, X.; Ramé, E.; Garoff, S. The effects of thin and ultrathin liquid films on dynamic wetting. Phys. Fluids 2004, 16, 287-297.

(68) Eggers, J.; Stone, H. A. Characteristic lengths at moving contact lines for a perfectly wetting fluid: the influence of speed on the dynamic contact angle. J. Fluid Mech. 2004, 505, 309-321. 